\title{
Überhang- und Ausgleichsmandate in Schleswig-Holstein: Unklares Wahlrecht und Reformvorschläge
}

\author{
Eric Linhart und Harald Schoen
}

Ein perfektes Wahlsystem gibt es nicht. Daher sieht sich auch die personalisierte Verhältniswahl im deutschen Wahlrecht, obgleich Modell für Wahlsystemreformen in verschiedenen Ländern, immer wieder Kritik ausgesetzt. Einen wichtigen Ansatzpunkt bildet dabei das mögliche und auch faktische Vorkommen von Überhangmandaten (siehe etwa die Beiträge von Waldemar Schreckenberger ${ }^{1}$, Joachim Behnke ${ }^{2}$, Eckhard Jesse $e^{3}$ oder Friedrich Pukelsheim und Sebastian Maier ${ }^{4}$, um nur einige zu nennen). Auch das Bundesverfassungsgericht wurde mehrfach mit der Problematik der Überhangmandate befasst und kam zu dem Schluss, dass diese grundsätzlich mit dem Grundgesetz vereinbar sind. Allerdings betonten die Richter etwa in ihrem Urteil vom 10. April 1997, dass nur eine moderate Anzahl von Überhangmandaten dem Grundcharakter der Wahl als Verhältniswahl nicht widerspreche und der Gesetzgeber darauf zu achten habe, dass sich die Zahl der Überhangmandate in Grenzen hält. Das Gericht sah die - nicht näher spezifizierte und schon gar nicht quantifizierte - Schwelle bei den bisherigen Bundestagswahlen noch nicht erreicht. ${ }^{5}$ Die Dresdner Nachwahl bei der Bundestagswahl 2005 rückte eine zweite Schwäche des deutschen Wahlrechts verstärkt ins Bewusstsein: das negative Stimmgewicht. Hierunter ist zu verstehen, dass eine Partei aufgrund des Berechnungsverfahrens der Sitzzuteilung einen Sitz verlieren kann, wenn sie eine zusätzliche Stimme erhält (unter Konstanthaltung der Stimmen für die übrigen Parteien). Auch zur Vereinbarkeit dieses Phänomens mit dem Grundgesetz wurde das Verfassungsgericht angerufen und gelangte im Juli 2008 zu dem Ergebnis, dass negatives Stimmgewicht nicht zulässig ist und das Bundeswahlgesetz bis spätestens zum 30. Juni 2011 so zu reformieren ist, dass es nicht mehr vorkommen kann. ${ }^{6}$ Dieses Ziel dürfte schwerlich vollständig erreichbar sein, da schon allein die Existenz einer Sperrklausel wie der Fünfprozenthürde zu einem negativen Stimmgewicht führen kann. Der bei der Bundestagswahl 2005 beobachtete Fall hatte jedoch eine andere Ursache, die mit der speziellen

1 Vgl. Waldemar Schreckenberger, Zum Streit über die Verfassungsmäßigkeit der Überhangmandate, in: ZParl, 26. Jg. (1995), H. 4, S. $678-683$.

2 Vgl. Joachim Behnke, Überhangmandate: Ein (behebbarer) Makel im institutionellen Design des Wahlsystems. Bamberger Beiträge zur Politikwissenschaft, Nr. I-6 2003; Joachim Behnke, Die Bombe tickt weiter: Das immer noch existierende Problem der Überhangmandate und mögliche Lösungen, in: Jürgen W. Falter / Oscar W. Gabriel / Bernhard Weßels (Hrsg.), Wahlen und Wähler. Analysen aus Anlass der Bundestagswahl 2002, Wiesbaden 2005, S. 459 - 483; Joachim Behnke, The Strange Phenomenon of Surplus Seats in the German Electoral System, in: German Politics, 16. Jg. (2007), H. 4, S. 496 - 517; Joachim Behnke, Überhangmandate bei der Bundestagswahl 2009. Eine Schätzung mit Simulation, in: ZParl, 40. Jg. (2009), H. 3, S. 620 - 636.

3 Vgl. Eckhard Jesse, Reformvorschläge zur Änderung des Wahlrechts, in: APuZ, B 52 (2003), S. 3 -11 .

4 Vgl. Friedrich Pukelsheim / Sebastian Maier, Parlamentsvergrößerung als Problemlösung für Überhangmandate, Pattsituationen und Mehrheitsklauseln, in: ZParl, 39. Jg. (2008), H. 2, S. 312 322.

5 Vgl. BVerfGE 95, S. 335.

6 Vgl. BVerfGE 121, S. 266.

Zeitschrift für Parlamentsfragen (ZParl), Heft 2/2010, S. 290 - 303 
Verrechnungsmethode über getrennte Landeslisten der Parteien zusammenhängt. ${ }^{7}$ Nun werden in der Bundesrepublik auch die meisten Landesparlamente nach der personalisierten Verhältniswahl gewählt, wobei keine Verrechnung über Landesteil-Listen vorgesehen ist; es existieren landesweite Listen. Negatives Stimmgewicht, das, wie im Urteil vom 3. Juli 2008 moniert, aus der zweistufigen Verteilung resultiert, ist auf Landesebene daher ausgeschlossen. Unter diesem Gesichtspunkt erscheint es folgerichtig, dass eine Reform des Bundeswahlgesetzes gefordert wird, die - ebenfalls nach personalisierter Verhältniswahl wählenden - Länder hiervon jedoch nicht betroffen sind.

Die jüngste Landtagswahl in Schleswig-Holstein demonstrierte jedoch eindrucksvoll, dass der Verzicht auf eine regionale Unterverteilung keine problemlose Stimmenverrechnung garantiert. Wie der Bundestag wurde der Kieler Landtag am 27. September 2009 gewählt. Sechs Parteien gelang der Einzug ins Parlament: der CDU (31,5 Prozent der Zweitstimmen), der SPD (25,4 Prozent), der FDP (14,9 Prozent), den Grünen (12,4 Prozent), der Linken (6,0 Prozent) sowie dem SSW (4,3 Prozent), der als Vertretung der dänischen und friesischen Minderheit von der Fünfprozent-Sperrklausel ausgenommen ist. Damit war jedoch noch nicht entschieden, mit wie vielen Mandaten die Parteien vertreten sein und welche Parteien die Regierung stellen würden. Die Ursache dafür liegt in einer Unklarheit des Landeswahlrechts.

\section{Interpretationsspielräume im schleswig-holsteinischen Wablrecht}

Laut Landesverfassung besteht der Schleswig-Holsteinische Landtag regulär aus 69 Mitgliedern, die nach dem Prinzip der personalisierten Verhältniswahl gewählt werden (Art. 10 Abs. 2 LV S-H). Die Bürger können zwei Stimmen abgeben: die Erststimme für einen Kandidaten im lokalen Wahlkreis, die Zweitstimme für eine Parteiliste. Auf der Basis der Erststimmen werden 40 Direktmandate vergeben; auf der Grundlage der Zweitstimmenergebnisse werden nach dem d'Hondtschen Höchstzahlverfahren mit Fünfprozent-Sperrklausel alle 69 Mandate verteilt. Anschließend werden die nach Erst- und Zweitstimmen vergebenen Mandate miteinander verrechnet. Sollte sich bei dieser Verrechnung herausstellen, dass eine Partei mehr Direkt- als Zweitstimmenmandate erhalten hat, entstehen Überhangmandate. Für diesen Fall schreibt Artikel 10 Abs. 2 LV S-H ein Gesetz vor, das Ausgleichsmandate vorsehen muss. Dementsprechend heißt es in $\$ 3$ Abs. 5 Landeswahlgesetz (LWG): „Ist die Anzahl der in den Wahlkreisen für eine Partei gewählten Bewerberinnen und Bewerber größer als ihr verhältnismäßiger Sitzanteil, so verbleiben ihr die darüber hinausgehenden Sitze (Mehrsitze). In diesem Fall sind auf die nach Absatz 3 Satz 2 und 3 noch nicht berücksichtigten Höchstzahlen so lange weitere Sitze zu verteilen und nach Absatz 3

7 Nach der Berechnung, wie viele Sitze einer Partei insgesamt zustehen (Oberverteilung), wird ermittelt, wie die Sitze auf die einzelnen Landeslisten zu verteilen sind (Unterverteilung). Dies hängt mit der Stärke der Partei in den einzelnen Ländern zusammen. Erhält eine Partei in einem Land A zusätzliche Stimmen, kann dies bedeuten, dass ihr zwar insgesamt nicht mehr Sitze gemäß der Oberverteilung zustehen, dass aber bei der internen Unterverteilung ein Sitz mehr auf die Landesliste A entfällt zuungunsten der Landesliste B. Hat die Partei in A Überhangmandate gewonnen, so ist der zusätzliche Sitz in A dadurch bereits abgedeckt. Sie erhält in A also keinen Sitz mehr, in B aber einen weniger, sofern sie nicht dort auch Überhangmandate besitzt. Insgesamt führen die Zusatzstimmen in A somit zu dem Verlust eines Sitzes für die Partei. 
zu besetzen, bis der letzte Mehrsitz durch den verhältnismäßigen Sitzanteil gedeckt ist. Die Anzahl der weiteren Sitze darf dabei jedoch das Doppelte der Anzahl der Mehrsitze nicht übersteigen. Ist die nach den Sätzen 1 bis 3 erhöhte Gesamtsitzzahl eine gerade Zahl, so wird auf die noch nicht berücksichtigte nächstfolgende Höchstzahl ein zusätzlicher Sitz vergeben. “Es sind also Ausgleichsmandate vorgesehen, falls Überhangmandate auftreten, ihre Zahl ist jedoch auf das „Doppelte der Anzahl der Mehrsitze“ beschränkt.

Diese Regelung enthält einen Interpretationsspielraum, nämlich dahingehend, wie viele Ausgleichsmandate höchstens gestattet sind. Beinhalten die „weiteren Sitze“ auch die Überhangmandate (Mehrsitze), oder sind mit ihnen allein die Ausgleichsmandate gemeint? In einer ersten Lesart bezieht sich das „Doppelte der Anzahl der Mehrsitze“ auf die Anzahl der Überhang- und Ausgleichsmandate. Eine zweite Interpretation sieht dagegen nur die Anzahl der Ausgleichsmandate auf das „Doppelte der Anzahl der Mehrsitze“ beschränkt. Erzielte eine Partei beispielsweise drei Überhangmandate, können diese in der ersten Deutung mit maximal drei weiteren Mandaten ausgeglichen werden, so dass zu den regulär 69 Mandaten höchstens sechs zusätzliche Mandate hinzukommen. In der zweiten Deutung wären maximal sechs Ausgleichsmandate zulässig, insgesamt kämen somit bis zu neun Sitze hinzu.

Diese Unklarheit gewann bei der Landtagswahl 2009 eine vorher nicht gekannte politische Brisanz, da die divergierenden Interpretationen zu gravierend verschiedenen Mehrheitsverhältnissen im Kieler Landeshaus führten. ${ }^{8}$ Nach dem Zweitstimmenergebnis (vgl. Tabelle 1, zweite Spalte) standen der CDU 23 Sitze zu, der SPD 19, der FDP elf, den Grünen neun, der Linkspartei vier und dem SSW drei. Im Ergebnis entfielen auf CDU und FDP zusammen 34 Mandate, also eines weniger, als für die parlamentarische Mehrheit erforderlich wäre (vgl. Tabelle 1, dritte Spalte). Grundlage für die Ermittlung der Sitzverteilung nach Zweitstimmen ist das Divisorverfahren nach d'Hondt. Hierbei wird ein geeigneter Divisor gesucht, durch den die absoluten Stimmenzahlen aller zu berücksichtigenden Parteien geteilt werden. Die abgerundeten Quotienten entsprechen der Sitzverteilung gemäß diesem Verfahren. Ein Divisor gilt dann als geeignet, wenn die Gesamtzahl der durch ihn ermittelten Mandate der Vorgabe (hier: 69) entspricht. Inhaltlich lässt sich der Divisor interpretieren als Anzahl der Wähler, die durch einen Parlamentarier vertreten werden. Geeignet sind hier alle Divisoren zwischen 21.068 und 21.454.9

Eine Alternative stellt die Anwendung des d'Hondt-Verfahrens als Höchstzahlverfahren dar, was zu demselben Ergebnis führt: Die Stimmenzahl der zu berücksichtigenden Parteien wird hierbei durch alle natürlichen Zahlen geteilt, und die Quotienten werden der Größe nach geordnet. Sind n Sitze zu vergeben, so spielen die n größten Quotienten eine Rolle; für jeden dieser Quotienten erhält die Partei, aus deren Stimmenzahl er resultiert, einen Sitz. Aus Tabelle 2 wird ersichtlich, dass gemäß dieser Herangehensweise der letzte der 69 zu vergebenden Sitze der 19. Sitz für die SPD ist. Der Zusammenhang mit der Anwendung

8 Bei den Landtagswahlen 1992 und 2000 traten bereits Überhangmandate und daher Ausgleichsmandate auf. Allerdings führten die unterschiedlichen Lesarten des Wahlgesetzes zu gleichen Schlussfolgerungen. In beiden Fällen erhielt die SPD sieben Überhangmandate, die durch sieben Ausgleichsmandate voll kompensiert wurden. Da auch ein großer Ausgleich abbricht, sobald eine Vollkompensation der Überhangmandate erreicht ist, stellte sich in beiden Fällen die Frage nach einer exakten Interpretation noch nicht.

9 Die Divisoren müssen nicht zwangsläufig ganzzahlig sein. Die exakten Werte bis auf zwei Nachkommastellen gerundet können Tabelle 2 entnommen werden. Gleiches gilt für im Folgenden genannte Divisoren. 


\begin{tabular}{|c|c|c|c|c|c|c|c|c|c|c|}
\hline \multirow[t]{2}{*}{ Tabelle 1: } & \multicolumn{10}{|c|}{$\begin{array}{l}\text { Mandatsverteilung im Schleswig-Holsteinischen Landtag in Abhängigkeit vom } \\
\text { Verrechnungsverfahren }\end{array}$} \\
\hline & 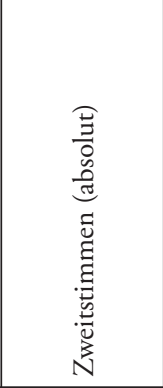 & 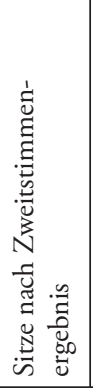 & 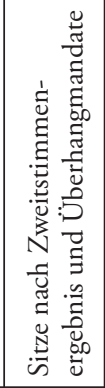 & 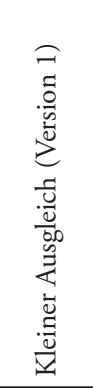 & 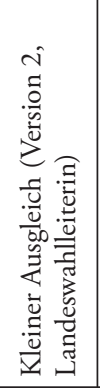 & 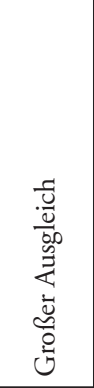 & 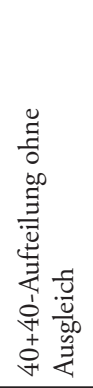 & 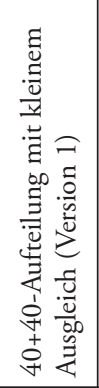 & 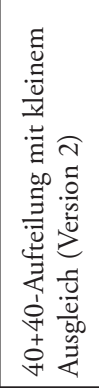 & 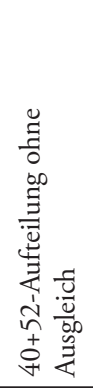 \\
\hline $\mathrm{CDU}^{\mathrm{a}}$ & 505.612 & 23 & $34(11)$ & $34(5)$ & $34(3)$ & $34(0)$ & $34(7)$ & $34(3)$ & $34(2)$ & $34(3)$ \\
\hline SPD & 407.643 & 19 & 19 & 23 & 25 & 28 & 22 & 25 & 26 & 25 \\
\hline FDP & 239.338 & 11 & 11 & 14 & 14 & 16 & 13 & 14 & 15 & 14 \\
\hline Grüne & 199.367 & 9 & 9 & 11 & 12 & 13 & 10 & 12 & 12 & 12 \\
\hline Linke & 95.764 & 4 & 4 & 5 & 6 & 6 & 5 & 6 & 6 & 6 \\
\hline SSW & 69.703 & 3 & 3 & 4 & 4 & 4 & 3 & 4 & 4 & 4 \\
\hline Sonstige & 85.979 & 0 & 0 & 0 & 0 & 0 & 0 & 0 & 0 & 0 \\
\hline Summe & 1.603 .374 & 69 & 80 & 91 & 95 & 101 & 87 & 95 & 97 & 95 \\
\hline $\begin{array}{l}\text { CDU } \\
\text { und FDP }\end{array}$ & 744.950 & 34 & 45 & 48 & 48 & 50 & 47 & 48 & 49 & 48 \\
\hline in Prozent & $46,5(49,1)^{\mathrm{b}}$ & 49,3 & 56,3 & 52,7 & 50,5 & 49,5 & 54,0 & 50,5 & 50,5 & 50,5 \\
\hline $\begin{array}{l}\text { Dissimi- } \\
\text { larität }\end{array}$ & & 5,54 & 10,97 & 6,33 & 5,69 & 5,78 & 7,56 & 5,69 & 5,65 & 5,69 \\
\hline \multicolumn{11}{|c|}{$\begin{array}{l}\text { a In Klammern unausgeglichene Überhangmandate. } \\
\text { b Stimmanteil von CDU und FDP ohne Berücksichtigung der Stimmen für Sonstige. } \\
\text { Quelle: Eigene Zusammenstellung. }\end{array}$} \\
\hline
\end{tabular}

des d'Hondt-Verfahrens als Divisorverfahren ist erkennbar, wenn man die Quotienten des 69. Mandats mit dem potenziellen 70. - einem 24. Sitz für die CDU - vergleicht. Die oben genannten Divisoren sind exakt diejenigen ganzen Zahlen, die zwischen den beiden Quotienten liegen.

Unabhängig von der Ermittlung des Zweitstimmenergebnisses gewann die CDU jedoch 34 der 40 Direktmandate, ihr fielen daher elf Überhangmandate zu. Diese Überhangmandate sind insofern als solche in Tabelle 2 erkennbar, als die Quotienten der entsprechenden Divisoren 24 bis 34 niedrigere Werte aufweisen als der letzte regulär zu vergebende Sitz. In der Folge hatten Christ- und Freidemokraten vor der Vergabe von Ausgleichsmandaten zusammen 45 von 80 Mandaten und verfügten damit über eine parlamentarische Mehrheit (vgl. Tabelle 1, dritte Spalte).

Bei der Verteilung der Ausgleichsmandate entschieden sich die Landeswahlleiterin und der Landeswahlausschuss für den kleinen Ausgleich. ${ }^{10}$ Ausgehend von den elf Überhangmandaten folgerten sie, dass insgesamt 22 „weitere“ Mandate (zusätzlich zu den 69 regulä-

10 Vgl. Endgültiges Ergebnis der Wahl zum Schleswig-Holsteinischen Landtag vom 27. September 2009. Bekanntmachung der Landeswahlleiterin vom 16. Oktober 2009 sowie den Nachtrag vom 22. Januar 2010. 


\begin{tabular}{|c|c|c|c|c|c|c|}
\hline \multicolumn{7}{|c|}{$\begin{array}{l}\text { Tabelle 2: Quotienten nach d'Hondt zur Ermittlung der Sitzverteilung im Schleswig- } \\
\text { Holsteinischen Landtag } 2009\end{array}$} \\
\hline Divisor & $\mathrm{CDU}$ & SPD & FDP & Grüne & Linke & SSW \\
\hline 1 & $505.612,00$ & $407.643,00$ & $239.338,00$ & $199.367,00$ & $95.764,00$ & $69.703,00$ \\
\hline 2 & $252.806,00$ & $203.821,50$ & $119.669,00$ & $99.683,50$ & $47.882,00$ & $34.851,50$ \\
\hline 3 & $168.537,33$ & $135.881,00$ & $79.779,33$ & $66.455,67$ & $31.921,33$ & $23.234,33$ \\
\hline 4 & $126.403,00$ & $101.910,75$ & $59.834,50$ & $49.841,75$ & $23.941,00$ & $17.425,75$ \\
\hline 5 & $101.122,40$ & $81.528,60$ & $47.867,60$ & $39.873,40$ & $19.152,80$ & $13.940,60$ \\
\hline 6 & $84.268,67$ & $67.940,50$ & $39.889,67$ & $33.227,83$ & $15.960,67$ & $11.617,17$ \\
\hline 7 & $72.230,29$ & $58.234,71$ & $34.191,14$ & $28.481,00$ & $13.680,57$ & $9.957,57$ \\
\hline 8 & $63.201,50$ & $50.955,38$ & $29.917,25$ & $24.920,88$ & $11.970,50$ & $8.712,88$ \\
\hline 9 & $56.179,11$ & $45.293,67$ & $26.593,11$ & $22.151,89$ & $10.640,44$ & $7.744,78$ \\
\hline 10 & $50.561,20$ & $40.764,30$ & $23.933,80$ & $19.936,70$ & $9.576,40$ & $6.970,30$ \\
\hline 11 & $45.964,73$ & $37.058,45$ & $21.758,00$ & $18.124,27$ & $8.705,82$ & $6.336,64$ \\
\hline 12 & $42.134,33$ & $33.970,25$ & $19.944,83$ & $16.613,92$ & $7.980,33$ & $5.808,58$ \\
\hline 13 & $38.893,23$ & $31.357,15$ & $18.410,62$ & $15.335,92$ & $7.366,46$ & $5.361,77$ \\
\hline 14 & $36.115,14$ & $29.117,36$ & $17.095,57$ & $14.240,50$ & $6.840,29$ & $4.978,79$ \\
\hline 15 & $33.707,47$ & $27.176,20$ & $15.955,87$ & $13.291,13$ & $6.384,27$ & $4.646,87$ \\
\hline 16 & $31.600,75$ & $25.477,69$ & $14.958,63$ & $12.460,44$ & $5.985,25$ & $4.356,44$ \\
\hline 17 & $29.741,88$ & $23.979,00$ & $14.078,71$ & $11.727,47$ & $5.633,18$ & $4.100,18$ \\
\hline 18 & $28.089,56$ & $22.646,83$ & $13.296,56$ & $11.075,94$ & $5.320,22$ & $3.872,39$ \\
\hline 19 & $26.611,16$ & $21.454,89$ & $12.596,74$ & $10.493,00$ & $5.040,21$ & $3.668,58$ \\
\hline 20 & $25.280,60$ & $20.382,15$ & $11.966,90$ & $9.968,35$ & $4.788,20$ & $3.485,15$ \\
\hline 21 & $24.076,76$ & $19.411,57$ & $11.397,05$ & $9.493,67$ & $4.560,19$ & $3.319,19$ \\
\hline 22 & $22.982,36$ & $18.529,23$ & $10.879,00$ & $9.062,14$ & $4.352,91$ & $3.168,32$ \\
\hline 23 & $21.983,13$ & $17.723,61$ & $10.406,00$ & $8.668,13$ & $4.163,65$ & $3.030,57$ \\
\hline 24 & $21.067,17$ & $16.985,13$ & $9.972,42$ & $8.306,96$ & $3.990,17$ & $2.904,29$ \\
\hline 25 & $20.224,48$ & $16.305,72$ & $9.573,52$ & $7.974,68$ & $3.830,56$ & $2.788,12$ \\
\hline 26 & $19.446,62$ & $15.678,58$ & $9.205,31$ & $7.667,96$ & $3.683,23$ & $2.680,88$ \\
\hline 27 & $18.726,37$ & $15.097,89$ & $8.864,37$ & $7.383,96$ & $3.546,81$ & $2.581,59$ \\
\hline 28 & $18.057,57$ & $14.558,68$ & $8.547,79$ & $7.120,25$ & $3.420,14$ & $2.489,39$ \\
\hline 29 & $17.434,90$ & $14.056,66$ & $8.253,03$ & $6.874,72$ & $3.302,21$ & $2.403,55$ \\
\hline 30 & $16.853,73$ & $13.588,10$ & $7.977,93$ & $6.645,57$ & $3.192,13$ & $2.323,43$ \\
\hline 31 & $16.310,06$ & $13.149,77$ & $7.720,58$ & $6.431,19$ & $3.089,16$ & $2.248,48$ \\
\hline 32 & $15.800,38$ & $12.738,84$ & $7.479,31$ & $6.230,22$ & $2.992,63$ & $2.178,22$ \\
\hline 33 & $15.321,58$ & $12.352,82$ & $7.252,67$ & $6.041,42$ & $2.901,94$ & $2.112,21$ \\
\hline 34 & $14.870,94$ & $11.989,50$ & $7.039,35$ & $5.863,74$ & $2.816,59$ & $2.050,09$ \\
\hline 35 & $14.446,06$ & $11.646,94$ & $6.838,23$ & $5.696,20$ & $2.736,11$ & $1.991,51$ \\
\hline 36 & $14.044,78$ & $11.323,42$ & $6.648,28$ & $5.537,97$ & $2.660,11$ & $1.936,19$ \\
\hline \multicolumn{7}{|c|}{$\begin{array}{l}\text { Grau } 10 \text { Prozent }=69 \text { reguläre Mandate. } \\
\text { Grau } 20 \text { Prozent }=11 \text { Überhangmandate der CDU. } \\
\text { Grau } 30 \text { Prozent }=11 \text { Ausgleichsmandate nach kleinem Ausgleich (Version 1). } \\
\text { Grau } 40 \text { Prozent }=9 \text { von maximal } 11 \text { weiteren Ausgleichsmandaten nach großem Ausgleich. } \\
\text { Grau } 55 \text { Prozent }=\text { Mandat im großen Ausgleich zur Erreichung einer ungeraden Anzahl. } \\
\text { Trennlinie }=91 \text { zu vergebende Mandate nach d'Hondt (kleiner Ausgleich, Version 2), aktuelle Sitzverteilung. } \\
\text { Gestrichelte Trennlinie = markiert die drei verbleibenden Überhangmandate der CDU sowie das Zusatzmandat } \\
\text { zum Erreichen einer ungeraden Anzahl an Sitzen (Linke). } \\
\text { Quelle: Eigene Zusammenstellung. }\end{array}$} \\
\hline
\end{tabular}


ren ohne Überhangmandate) entstehen müssten. An dieser Stelle kommt eine weitere Regelungslücke zum Tragen, die wieder Interpretationsspielraum lässt. Fraglich ist, nach welchem genauen Prozedere die Ausgleichsmandate zu vergeben sind. Im Gegensatz zu der Frage ,großer oder kleiner Ausgleich' war dieser Punkt nicht in der öffentlichen Diskussion wahrnehmbar und ist - soweit ersichtlich - auch nicht Gegenstand der Debatte über eine Wahlrechtsänderung. Gleichwohl beeinflusst auch die technische Ausgestaltung der Vergabe von Ausgleichsmandaten die Sitzverteilung nicht unerheblich, wie in Tabelle $1 \mathrm{zu}$ sehen ist.

Eine erste Möglichkeit (Version 1) geht von den 69 regulären sowie den elf Überhangmandaten für die CDU aus und sucht nun nach einem Modus, die elf Ausgleichsmandate $^{11}$ auf die übrigen im Parlament vertretenen Parteien zu verteilen. Nach dem d'HondtVerfahren ist demnach ein Divisor zu suchen, der auf $91 \mathrm{zu}$ verteilende Mandate abzielt, die Mandatszahl für die CDU aber unabhängig vom entsprechenden Quotienten auf 34 fixiert. Solche Divisoren sind alle Zahlen zwischen 16.986 und 17.095. Diese gelten allerdings nicht für das fixierte Ergebnis der CDU. Fünf ihrer Direktmandate sind nach diesem Ausgleich nicht kompensierte Überhangmandate. Von den elf Ausgleichsmandaten entfallen auf die SPD vier, auf die FDP drei, auf die Grünen zwei und auf Linke und SSW je eines (vgl. Tabelle 1, fünfte Spalte). Intuitiv plausibler ist dieses Vorgehen, wenn das d'HondtVerfahren wie oben beschrieben als Höchstzahlverfahren angewendet wird, was zu demselben Ergebnis führt: Die 69 regulären und die elf Überhangmandate seien gegeben, dann sind unter den verbleibenden Quotienten die elf größten auszuwählen und die entsprechenden Parteien mit Ausgleichsmandaten zu versehen (vgl. Tabelle 2). Unabhängig vom Rechenweg erhielten unter dieser Lösung CDU und FDP zusammen 48 von 91 Sitzen und verfügten somit über eine Parlamentsmehrheit.

Die Wahlleiterin entschied sich für eine alternative Methode, den kleinen Ausgleich in die Tat umzusetzen (Version 2, Landeswahlleiterin). Sie verteilte die Sitze, indem sie das d'Hondt-Verfahren nicht nur auf 69, sondern auf 91 Mandate anwandte. Bei dieser größeren Mandatszahl sind alle ganzen Zahlen zwischen 15.961 und 16.305 geeignete Divisoren; es ergaben sich für die CDU 31 Zweitstimmensitze, so dass drei CDU-Überhangmandate nicht von Ausgleichsmandaten gedeckt sind. Diese Verteilungsprozedur führt für die SPD zu 25, für die FDP zu 14, für die Grünen zu zwölf, für die Linke zu fünf und für den SSW zu vier Sitzen. Ungeachtet der 31 der CDU nach dieser Rechnung zustehenden Sitze bleiben alle Direktmandate erhalten, so dass die CDU 34 Abgeordnete stellt. Um die im Landeswahlrecht geforderte ungerade Anzahl an Sitzen herzustellen, ist ein weiteres Mandat notwendig, das gemäß der d'Hondt-Methode an die Linke fällt. ${ }^{12}$ Damit verfügen CDU und FDP zusammen über 48 von 95 Mandaten im Kieler Landeshaus, und damit über eine parlamentarische Mehrheit (vgl. Tabelle 1, sechste Spalte). Streng genommen wider-

11 Korrekt ist, dass maximal elf weitere Mandate zu vergeben sind. Grundsätzlich ist nicht ausgeschlossen, dass die Überhangmandate auch durch weniger weitere Mandate ausgeglichen werden können. Dies ist hier nicht der Fall, weshalb wir beim kleinen Ausgleich im Folgenden vom Maximum elf ausgehen.

12 Gemäß der Bekanntmachung vom 16. Oktober 2009 wurde dieses Mandat der FDP zugesprochen. Eine Nachzählung der Stimmen im Stimmbezirk Husum 3 am 22. Januar 2010 ergab, dass bei dem ursprünglich verkündeten Wahlergebnis der Linken 32 Zweitstimmen zu wenig zugesprochen wurden. Mit diesen zusätzlichen Stimmen stieg der sechste Divisor der Linken von 15.955,33 auf 15.960,67 und war somit größer als der 15. Divisor der FDP $(15.955,87)$. 
spricht dieses Ergebnis der Intention eines kleinen Ausgleichs: Nimmt man die Begrenzung auf insgesamt 91 Mandate ernst, so ist eine Zuteilung von 95 Mandaten nicht zulässig. Dass ein kleiner Ausgleich möglich ist, der die Begrenzung einhält, zeigt Version 1.

Im Gegensatz zu den beiden Varianten des kleinen Ausgleichs sind im großen maximal 22 Ausgleichsmandate zu vergeben sowie gegebenenfalls ein zusätzliches Mandat, um eine ungerade Sitzanzahl herzustellen. Insgesamt umfasste ein dieser Lesart folgender Landtag somit höchstens 103 Abgeordnete. Der 34. CDU-Sitz, also das letzte auszugleichende Überhangmandat, wäre aber schon bei einer Landtagsgröße von 100 Abgeordneten ein reguläres Mandat. Nach der Vergabe eines 101. Mandats an die SPD zur Herstellung der ungeraden Sitzzahl wäre der Ausgleichsprozess beendet, ohne dass die Maximalzahl an Ausgleichsmandaten ausgeschöpft werden müsste. Aus Tabelle 2 geht hervor, dass ein 102. zu vergebendes Mandat wieder an die CDU ginge, also kaum als Ausgleichsmandat für die CDU-Überhangmandate interpretiert werden könnte. Da bei einem großen Ausgleich alle Überhangmandate kompensiert werden, kommen Unterschiede in der technischen Durchführung des Ausgleichs anders als beim kleinen Ausgleich (Version 1 und 2) nicht zum Tragen. Im Endergebnis hätten nach dieser Ausgleichsprozedur die CDU 34, die SPD 28, die FDP 16, die Grünen 13, die Linkspartei sechs und der SSW vier Mandate. CDU und FDP hätten zusammen 50 von 101 Sitzen und wären somit ohne Mehrheit im Landesparlament.

Die verschiedenen Interpretationen der Klausel im Wahlgesetz führen also zu unterschiedlichen Mehrheitsverhältnissen im Landtag. Daher kann es auch kaum überraschen, dass nach der Entscheidung der Landeswahlleiterin und des Landeswahlausschusses öffentliche Diskussionen und rechtliche Auseinandersetzungen über die Auslegung des Landeswahlgesetzes einsetzten. Die Fraktion von Bündnis 90/Die Grünen und die SSW-Abgeordneten strengten eine Normenkontrollklage vor dem Landesverfassungsgericht an, verbunden mit einem Antrag auf einstweilige Anordnung gegen die Anwendung des strittigen Satzes 3 in $\$ 3$ Abs. 5 LWG. Diesen Antrag wies das Landesverfassungsgericht jedoch als unzulässig zurück; die Entscheidung in der Sache steht noch aus. So konnte sich der neue Landtag am 27. Oktober 2009 auf der Basis der von der Landeswahlleiterin gewählten und vom Landeswahlausschuss bestätigten Lesart ${ }^{13}$ konstituieren und Peter Harry Carstensen, der Kandidat der christlich-liberalen Koalition, wieder zum Ministerpräsidenten gewählt werden, und zwar mit 50 von 95 Stimmen - also einer Stimme mehr, als CDU und FDP nach dem kleinen Ausgleich Sitze im Landtag zustehen. ${ }^{14}$

\section{Reformnotwendigkeit und Reformmöglichkeiten}

Diese Landtagswahl veranschaulicht sehr gut, dass Wahlrechtsfragen nicht Nebensächlichkeiten sind, sondern die parlamentarische Machtverteilung entscheidend beeinflussen können. Zudem demonstriert sie, wie schädlich unklare, unterschiedlichen Auslegungen zu-

$13 \mathrm{Zu}$ diesem Zeitpunkt zogen 15 Abgeordnete der FDP, aber nur fünf der Linkspartei in den Landtag ein (vgl. Fußnote 12).

14 Vgl. auch den Beitrag von Patrick Horst in diesem Heft. Die parlamentarische Mehrheit von Christ- und Freidemokraten droht nicht während der Wahlperiode wegen des Ausscheidens von Abgeordneten auf Überhangmandaten abzuschmelzen, da auch in diesem Fall Listenbewerber nachrücken würden. 
gängliche Regeln im Wahlrecht sind. Sie beeinträchtigen einen Konsens über den Ausgang einer Wahl und die Zusammensetzung von Parlament und Regierung. Die Probleme werden in Schleswig-Holstein dadurch nicht abgemildert, dass die Auslegung der Landeswahlleiterin in Einklang mit früheren Lesarten bei der Anwendung von kommunalem Wahlrecht steht und dort durch Gerichte bestätigt wurde. Für eine rechtspolitische Einschätzung der Problematik halten sich die Verwaltungsgerichte nicht zuständig. ${ }^{15}$ Regierungen, die aufgrund umstrittener Regeln ins Amt gelangen, besitzen eine anfechtbare Legitimation und können sich nicht auf eine unumstrittene Legitimität ihres Handelns berufen. ${ }^{16}$ Die unklare Regel, die der Forderung nach Transparenz und Klarheit von Wahlsystemen ${ }^{17}$ nicht entspricht, sollte daher durch eine eindeutige Vorschrift ersetzt werden. Mindestens muss der Landtag den umstrittenen Passus im Landeswahlrecht so formulieren, dass keinerlei Interpretationsspielräume mehr bleiben. Neben der im Vordergrund der Debatte stehenden Frage, ob die „weiteren“ Mandate die Überhangmandate einschließen oder nicht, ist auch eindeutig zu regeln, wie ein Ausgleich technisch vorzunehmen ist, sollte er in einer reformierten Form des Wahlrechts noch eine Rolle spielen.

Aus dieser vermutlich konsensuellen rechtsstaatlichen Minimalforderung folgt allerdings keine eindeutige Handlungsanweisung, denn die monierte Regelung könnte im Sinne des kleinen oder des großen Ausgleichs klargestellt werden. Eine Entscheidung für eine konkrete Novellierung erfordert es daher, sich Kenntnis darüber zu verschaffen, welchen Beitrag verschiedene Lösungsansätze leisten, mit dem Wahlsystem verfolgte Zielsetzungen zu erreichen. Da die Ziele nicht ohne weiteres vereinbar sind ${ }^{18}$, sondern in Konflikt zueinander treten können, ist schließlich zu klären, in welcher Gewichtung die Ziele verfolgt werden sollen. Letzteres ist eine normative, genuin politische Wertentscheidung, die in der politischen Auseinandersetzung zu treffen ist; hier soll versucht werden, Implikationen verschiedener Reformvorschläge aufzuzeigen und dadurch zu Klarheit in der Diskussion beizutragen.

Nachfolgend werden mögliche Reformen unter drei Gesichtspunkten diskutiert. Mit der Repräsentation und der Konzentration werden zwei Zielsetzungen berücksichtigt, die aus Katalogen mit Maßstäben zur Beurteilung von Wahlsystemen nicht wegzudenken sind. ${ }^{19}$ Auch im Wahlrecht des Landes Schleswig-Holstein haben sie ihren Niederschlag gefunden: In der Verfassung heißt es, dass ein Wahlsystem einzurichten sei, „das die Persönlichkeitswahl mit den Grundsätzen der Verhältniswahl verbindet“. Das Repräsentationsziel fordert eine möglichst gute Annäherung der Sitz- an die Zweitstimmenverteilung. Die Konzentrationsfunktion von Wahlsystemen ist hingegen darauf gerichtet, regierungsfähige Mehrhei-

15 Siehe etwa Schleswig-Holsteinisches Oberverwaltungsgericht, Ausgleichsmandatsregelung Kommunalwahlen SH, Urteil vom 15. September 2009 (2 LA 35/09).

16 Ein eindrückliches Beispiel dafür lieferte die US-amerikanische Präsidentschaftswahl im Jahr 2000. Siehe hierzu etwa Bruce Ackerman (Hrsg.), Bush v. Gore: The Question of Legitimacy, New Haven 2002; Anthony Simon Laden, Democratic Legitimacy and the 2000 Election, in: Law and Philosophy, 21. Jg. (2002), H. 2, S. $197-220$.

17 Vgl. Dieter Nohlen, Wahlrecht und Parteiensystem, Opladen 2000, S. 159; Eckhard Jesse, Wahlsysteme und Wahlrecht, in: Oscar W. Gabriel / Sabine Kropp (Hrsg.), Die EU-Staaten im Vergleich. Strukturen, Prozesse, Politikinhalte, Wiesbaden 2008, S. 299 - 322, S. 317.

18 Vgl. zum Antagonismus der Repräsentations- und der Konzentrationsfunktion etwa Eric Linhart, Mögliche Auswirkungen von Grabenwahlsystemen in der Bundesrepublik Deutschland. Theoretische Überlegungen und Simulationen, in: ZParl, 40. Jg. (2009), H. 3, S. 637 - 660.

19 Vgl. Dieter Nohlen, a.a.O. (Fn. 17); Eckhard Jesse, a.a.O. (Fn. 17). 
ten zu schaffen. Beide Ziele sind offenkundig nicht ohne weiteres gleichzeitig zu erreichen. Als drittes wird der Imperativ eines kleinen Parlaments einbezogen, das heißt eines Parlaments, das möglichst nur aus der gesetzlich festgelegten Anzahl von Abgeordneten besteht. Ein solches Ziel ist nicht aus Anforderungen an Wahlsysteme ableitbar, entsteht aber aus dem verständlichen Wunsch, Kosten einzusparen. Hält man einen Landtag mit 69 Abgeordneten für groß genug, um arbeitsfähig zu sein und die Bevölkerung ausreichend zu repräsentieren, so gibt es aus ökonomischer Sicht keinen Grund, sich ein größeres Parlament zu leisten, das höhere Kosten verursacht. Für diese Zielsetzung sprechen unter anderem die 2003 per Verfassungsänderung beschlossene Verkleinerung des Landtages und die Tatsache, dass CDU und FDP in ihrer Koalitionsvereinbarung nach der Landtagswahl 2009 eine Wahlrechtsreform anstrebten mit dem Ziel, „eine Überschreitung der in der Landesverfassung vorgesehenen Landtagsmandate zu vermeiden " 20 .

In nuce spiegeln die Regelungen des Landeswahlrechts zu den Überhangmandaten diese Trias nicht immer zu vereinbarender Zielsetzungen wider. Die Tatsache, dass überhaupt Überhangmandate entstehen können, lässt sich als Indiz dafür werten, dass der Verfassungsgesetzgeber einen Konzentrationseffekt - und eine Vergrößerung des Parlaments - zumindest in Kauf nimmt. Anders als etwa auf Bundesebene sind nicht die Hälfte der Sitze Direktmandate und die andere Hälfte Listenmandate; die 40 Direktmandate bei den Landtagswahlen betragen etwa 58 Prozent der regulären Mandate. Dies begünstigt das Entstehen von Überhangmandaten und verstärkt somit dieses Indiz. Zugleich schreibt die Verfassung Ausgleichsmandate vor, was als Ausfluss des Repräsentationsimperativs zu lesen ist. Außerdem wird eine obere Grenze der Anzahl der Ausgleichsmandate definiert. Dies kann man zum einen als Einhegung des Repräsentationsziels zugunsten der Konzentrationsfunktion interpretieren. Zum anderen kommt darin - und im Gegensatz zu den beiden anderen Vorschriften - der Imperativ zum Ausdruck, den Landtag nicht unbegrenzt anwachsen zu lassen.

Wie könnten nun Reformansätze aussehen? Eine „kleine“ Lösung zur Klarstellung könnte darin bestehen, den Interpretationsspielraum in $₫ 3$ Abs. 5 S. 3 LWG zugunsten einer eindeutigen Regelung im Sinne des kleinen oder großen Ausgleichs zu beseitigen und die technische Umsetzung des Ausgleichs festzulegen. Der kleine Ausgleich läuft - wie 2009 zu sehen war - darauf hinaus, dass die durch Überhangmandate entstehende Verzerrung der Sitzverteilung im Vergleich zur Stimmenverteilung zwar abgemildert wird. Sie kann aber nicht in jedem Fall beseitigt werden. So läge die Dissimilarität ${ }^{21}$, also die Unähnlichkeit zwischen Stimm- und Sitzverteilung, ohne Überhangmandate bei 5,54, mit Überhangmandaten fast doppelt so hoch bei 10,97. Der kleine Ausgleich reduziert diesen Wert auf 6,33 beziehungsweise 5,69. Eine Konsequenz daraus ist, dass 2009 mit CDU und FDP

20 Christlich-Demokratische Union (CDU) und Freie Demokratische Partei (FDP) Schleswig-Holstein, Koalition des Aufbruchs. Koalitionsvertrag zwischen der Christlich-Demokratischen Union (CDU) und der Freien Demokratischen Partei (FDP) Schleswig-Holstein für die 17. Legislaturperiode des Schleswig-Holsteinischen Landtags, Kiel 2009, S. 43.

21 Die in Tabelle 1 ausgewiesenen Werte für die Dissimilarität beruhen auf dem Loosemore-HanbyIndex. Der Index misst für alle Parteien die Differenzen zwischen prozentualem Stimm- und Sitzanteil, summiert deren Absolutwerte auf und teilt die Summe durch 2. Vgl. John Loosemore I Victor J. Hanby, The Theoretical Limits of Maximum Distortion: Some Analytic Expressions for Electoral Systems, in: British Journal of Political Science, 1. Jg. (1971), H. 4, S. $467-477$. 
zwei Parteien die Parlamentsmehrheit erringen konnten, obwohl sie weniger Stimmen für sich verbuchten als die parlamentarischen Oppositionsparteien. ${ }^{22}$ Entstünden weniger Überhangmandate, könnte auch der kleine Ausgleich zu einer vollständigen Anpassung der Mandats- an die Stimmenverteilung führen. Hätte die CDU 2009 nicht mehr als drei Überhangmandate erzielt, wäre dies gelungen.

Die Implikationen des kleinen Ausgleichs für die Erreichung des Proportionalitätsziels hängen daher entscheidend von der künftigen Entwicklung des Wahlverhaltens ab. Sollte die CDU weiterhin die übergroße Mehrheit der Direktmandate erringen, aber deutlich weniger als die Hälfte oder auch nur 40 Prozent der Zweitstimmen gewinnen, ist nicht damit zu rechnen, dass dem kleinen Ausgleich eine vollständige Proportionalisierung der Sitzverteilung gelingen wird. Das heißt, das Repräsentationsziel wäre wohl vor allem dann zu erreichen, wenn die CDU deutlich höhere Zweitstimmenanteile erreichte oder weniger Direktmandate gewönne, das heißt wenn sich die SPD mehr Wahlkreise sichern könnte. Beides scheint nicht ausgeschlossen, ist aber keineswegs sicher. Unter dem Gesichtspunkt des Repräsentationsziels schneidet der kleine Ausgleich somit eher schlecht ab.

Zugleich hat die Wahl 2009 eindrucksvoll demonstriert, dass der kleine Ausgleich keineswegs ein kleines Parlament bedeutet. Anstelle von regulär 69 wurden 95 Mandate vergeben, so dass die Zahl der Sitze um mehr als 37 Prozent anwuchs. Ein kleiner Ausgleich mit konsequent angewendeter Obergrenze (Version 1) führte zu einem Parlament mit 91 Sitzen. Damit wäre das Parlament zwar nicht so stark angewachsen, aber doch noch immer um beinahe ein Drittel größer als in der Verfassung vorgesehen. Insgesamt ist daher dem kleinen Ausgleich in seinen beiden Varianten ein eher schlechtes Zeugnis auszustellen.

Unter dem Gesichtspunkt der Konzentrations- und Regierungsbildungsfunktion kann er aber auf den ersten Blick einen eindeutigen Pluspunkt verbuchen, denn im Gegensatz zum großen Ausgleich ermöglichte er die Bildung einer klassischen „kleinen“ Koalition aus einer Volkspartei und einem kleinen Bündnispartner. In dem konkreten hier untersuchten Fall spielt dabei keine Rolle, wie der kleine Ausgleich technisch in die Tat umgesetzt wird. Grundsätzlich sieht Version 1 aber weniger Ausgleichsmandate vor, so dass eine stärkere Konzentration erwartbar ist, verbunden mit einer größeren Wahrscheinlichkeit, dass solche kleinen Koalitionen parlamentarische Mehrheiten erhalten.

Diese Bewertung ist allerdings in zweierlei Hinsicht zu relativieren. Zum einen ist eine kleine Koalition kein Wert an sich. Zwar dürfte außer Frage stehen, dass eine Große Koalition nicht zum Regelfall werden sollte, da sie das Widerspiel zwischen der Regierung und einer starken Opposition aushebelt. Doch heißt das nicht, dass Regierungsbündnisse aus mehr als zwei Parteien notwendigerweise weniger stabil und gestaltungsfähig sind als klassische kleine Koalitionen. Zum anderen könnte ein Wandel der Politischen Kultur im Lande dazu führen, dass Bündnisse aus drei und mehr Parteien weniger exotisch anmuten und ihr Zustandekommen nicht von politischen oder persönlichen Barrieren zwischen potentiellen Partnern verhindert wird. Unter den gegenwärtigen politischen Bedingungen schneidet der kleine Ausgleich somit im Hinblick auf die Regierungsbildungsfunktion vergleichsweise gut ab, doch muss das in Zukunft nicht so bleiben. Betrachtet man allerdings die Ermöglichung kleiner Koalitionen - unabhängig von konkreten Regierungsbildungsprozessen - als

22 Dieses Phänomen ist nicht neu: Bei der Wahl 1992 errang die SPD sämtliche Direktmandate und dank der Überhangmandate eine Mandatsmehrheit im Landtag, obwohl die parlamentarischen Oppositionsparteien mehr Stimmen als die Sozialdemokraten erzielt hatten. 
Indiz für die Wirksamkeit des Konzentrationseffekts, so ist der kleine Ausgleich - in beiden Versionen - dem großen überlegen.

Die Bewertung des großen Ausgleichs ähnelt in gewissem Maße einem Spiegelbild des Urteils über den kleinen. Wie aus Tabelle 1 hervorgeht, ist der große Ausgleich mit zwei gravierenden Nachteilen behaftet: Zum einen würde das Parlament auf 101 Mandate anwachsen und wäre damit beinahe anderthalbmal so groß wie in der Verfassung vorgesehen. Allerdings wären das „nur“ sechs Sitze mehr, als der Landtag nach dem kleinen Ausgleich in der Interpretation der Wahlleiterin umfasst. Zum zweiten verfügten CDU und FDP nach dieser Verteilungsprozedur zusammen über 50 Mandate und besäßen daher keine eigene Mehrheit. Der große Ausgleich zeigt insoweit keinen Konzentrationseffekt und stattet keine der möglichen kleinen Koalitionen mit einer parlamentarischen Mehrheit aus. Zumindest unter den momentanen politischen Bedingungen ist das als eine Schwäche zu werten. Zudem gelingt es mithilfe des großen Ausgleichs zumindest in dem konkreten Fall nicht, die Dissimilarität zwischen Zweitstimmen und Mandaten wesentlich zu senken. Mit einem Wert von 5,78 liegt sie zwar unter der des kleinen Ausgleichs gemäß Version 1, aber sogar leicht über der des kleinen Ausgleichs nach Version 2.

In der Summe sorgt der große Ausgleich für eine ähnlich proportionale Sitzverteilung wie der kleine, ermöglicht aber keine kleine Koalition, während dem kleinen Ausgleich letzteres gelingt, nicht jedoch ersteres. Alle hier diskutierten Möglichkeiten führen schließlich zu Parlamenten, die - mehr oder weniger deutlich - die regulär anvisierte Größe von 69 Abgeordneten übersteigen.

\section{Alternativen zur Präzisierung der Ausgleichsmandate-Regelung}

Die bisher diskutierten Lösungsansätze gleichen einander auch insofern, als sie die Entstehung von Überhangmandaten akzeptieren und die Klausel im Landeswahlgesetz zur Vergabe von Ausgleichsmandaten zu präzisieren suchen. Reformen des Wahlrechts könnten jedoch auch bei der Entstehung von Überhangmandaten ansetzen - und im Erfolgsfall eine Präzisierung des unklaren Passus im Landeswahlgesetz entbehrlich machen oder eine entsprechende Novellierung faktisch ihrer Wirkung berauben. Will man bei gegebenem Wahlverhalten und unter Beibehaltung einer um Elemente einer Persönlichkeitswahl ergänzten Verhältniswahl das Auftreten von Überhangmandaten vermeiden, erweist sich der Anteil der Direktmandate an der Zahl der regulären Sitze als die entscheidende Stellgröße. Sinkt der Anteil der Wahlkreismandate, werden Überhangmandate weniger wahrscheinlich - und es wird wahrscheinlicher, dass die gesetzliche und die tatsächliche Zahl der Abgeordneten zusammenfallen. Dieser Reformansatz mag in Schleswig-Holstein insoweit zusätzlich attraktiv wirken, als hier der Anteil der Direktmandate wie oben beschrieben mit 40 von 69 deutlich über dem entsprechenden Wert von 50 Prozent im Bund und in einigen anderen Bundesländern liegt. ${ }^{23}$

Um den Anteil der Direktmandate zu senken, kann man diese reduzieren oder die Gesamtzahl der Mandate bei konstanter Anzahl von Direktmandaten vergrößern. Erhöhte

23 Beispiele sind Brandenburg, Hessen, Sachsen und Thüringen sowie - bis auf einen Sitz - Mecklenburg-Vorpommern, Rheinland-Pfalz und Sachsen-Anhalt. In Nordrhein-Westfalen und Niedersachsen allerdings liegt der Anteil der Direktmandate mit 128 von 181 Mandaten (71 Prozent) beziehungsweise 87 von 135 Sitzen (64 Prozent) noch höher als in Schleswig-Holstein. 
man beispielsweise die Sitzanzahl auf 80, um 40 Direkt- ebenso viele Listenmandate gegenüberzustellen, wäre es bei der Landtagswahl 2009 zu folgender Sitzverteilung nach Zweitstimmen gekommen: CDU 27, SPD, 22, FDP 13, Grüne zehn, Linke fünf und SSW drei (vgl. Tabelle 1, achte Spalte). ${ }^{24}$ Im Ergebnis wären immer noch Überhangmandate zugunsten der CDU entstanden, allerdings nicht mehr elf, sondern nur noch sieben. Im Vergleich zum derzeitigen Anteil von 40 an 69 Direktmandaten ist die Repräsentationsfunktion zwar besser erfüllt (niedrigere Dissimilarität); die nicht auf einer Stimmenmehrheit beruhende Parlamentsmehrheit von CDU und FDP ist aber auch hier zu erkennen. Als Alternative zu einer Ausgleichsmandate-Regelung scheint eine Reform des Anteils von Direkt- zu Listenmandaten nicht ausreichend, um das Ziel der Repräsentation zu erreichen. Beide Versionen des kleinen Ausgleichs spiegeln das Wahlergebnis besser in der Sitzverteilung wider, und die künstliche Mehrheit fällt dort nicht ganz so deutlich aus wie in der Simulation. Die Berechnung mit 40 Listenmandaten besitzt aber den Vorteil eines kleineren Parlaments, als bei den verschiedenen Möglichkeiten des Ausgleichs vorkommen.

Eine Erhöhung des Anteils an Listenmandaten ist grundsätzlich ebenfalls mit einer Ausgleichsmandate-Regelung kombinierbar. Nach Anwendung des kleinen Ausgleichs (vgl. Tabelle 1, neunte und zehnte Spalte) würden drei beziehungsweise zwei ungedeckte Überhangmandate der CDU verbleiben, mit der Folge, dass auch in diesen Fällen CDU und FDP zusammen über 48 von 95 respektive 49 von 97 Sitzen und damit über eine Parlamentsmehrheit verfügten. Immerhin finden sich hierfür Dissimilaritätswerte, die der einer Sitzverteilung ohne Überhangmandate (Spalte 3) nahe kommen. Diese Lösung ist insofern positiv zu bewerten, als sie in diesem konkreten Fall sowohl die Konzentrations- als auch die Repräsentationsfunktion vergleichsweise gut erfüllt. Ein großer Ausgleich würde zu demselben Ergebnis führen wie der große Ausgleich auf Basis der aktuellen Aufteilung (vgl. Tabelle 1, siebte Spalte). Der Ansatz einer (relativen) Erhöhung der Zahl der Listenmandate vermindert die Zahl der Überhangmandate, verhindert deren Entstehung aber nicht vollständig. Strebte man dieses Ziel an, müsste man den Anteil der Direktmandate wesentlich stärker absenken. Geht man von der Verteilung der 40 Direktmandate bei der Landtagswahl 2009 aus, müsste der Landtag regulär hundert Sitze umfassen, damit kein einziges Überhangmandat entstünde. In diesem Fall wären die Mandate weitgehend proportional zu den Zweitstimmenanteilen verteilt. CDU und FDP hätten zusammen 50 Mandate, also keine eigene Parlamentsmehrheit. Das Repräsentationsziel würde erreicht, nicht jedoch eine klare Mehrheit für eine kleine Koalition. Und das Parlament würde noch mehr Abgeordnete umfassen, als es nach der Wahl 2009 tatsächlich der Fall ist.

Wollte man Überhangmandate vermeiden und die tatsächliche Sitzanzahl der gesetzlichen von 69 anpassen, böte sich daher der Weg an, bei konstanter Landtagsgröße die Zahl der Direktmandate zu senken. In diese Richtung zielt etwa der Gesetzentwurf der Fraktion von Bündnis 90/Die Grünen im Schleswig-Holsteinischen Landtag, der nur noch 30 Direktmandate vorsieht, also gut 43 Prozent der regulären Gesamtmandate. Die Wirkungen einer solchen Reform hängen nicht zuletzt vom exakten Zuschnitt der neuen Wahlkreise ab und lassen sich deshalb nur schwer exakt beziffern. Rechnet man den Anteil an Direktmandaten von 43 Prozent hoch auf die derzeitig zu vergebenden 40 Direktmandate, so müssten 92 Sitze insgesamt vergeben werden. Aus Tabelle 1 (elfte Spalte) geht wieder die entspre-

24 Wir treffen bei dieser wie bei allen folgenden Simulationen die Annahme, dass die veränderten Regeln das Wahlverhalten nicht beeinflusst hätten. 
chende Sitzverteilung hervor. In diesem Fall entspricht die Sitzaufteilung exakt der momentanen Ausgestaltung des Wahlrechts nach Lesart der Wahlleiterin mit den oben bereits diskutierten entsprechenden Konsequenzen, insbesondere einer künstlichen schwarz-gelben Mehrheit. Es spricht einiges dafür, dass in einem kleineren Landtag mit ähnlicher Aufteilung von Direkt- und Listenmandaten keine strukturell anderen Ergebnisse zu erwarten sind.

Um Überhangmandate sicher zu vermeiden und damit einen Landtag mit 69 Sitzen zu garantieren, müsste die Zahl der Direktmandate daher noch deutlicher reduziert werden. Auch dieser Schritt hätte seinen Preis: Die Wahlkreise würden wesentlich größer. Bei 40 Direktmandaten entfallen auf einen durchschnittlichen Wahlkreis knapp 50.000 Wahlberechtigte, bei 30 Direktmandaten sind es bereits rund 65.000. Es resultierte also eine schlechtere Relation zwischen Repräsentierten und Repräsentanten. Allerdings wäre das Zahlenverhältnis in Schleswig-Holstein damit immer noch günstiger als in einigen anderen Bundesländern. ${ }^{25}$

\section{Reformvorschläge aus Sicht der Parteien}

Die gegenwärtige Regelung von Ausgleichsmandaten im Landeswahlgesetz ermöglicht unterschiedliche Interpretationen, die zu unterschiedlichen Sitzverteilungen im Parlament führen - mit dem Ergebnis von (mindestens) drei Größen des Landtags mit unterschiedlichen Mehrheitsstrukturen. Als wichtigste Forderung an eine Wahlrechtsreform hat daher die Schaffung von Verfahrenssicherheit zu gelten. Dieser Imperativ bezieht sich sowohl auf die Frage, ob die bisher nicht näher spezifizierten „weiteren Mandate“ im Sinne eines kleinen oder großen Ausgleichs zu verstehen sind, als auch auf die Spezifikation der technischen Umsetzung eines Ausgleichs. ${ }^{26}$

Weitere Reformschritte hängen von der normativen Bewertung der drei mit Wahlsystemen verfolgbaren Ziele - (proportionale) Repräsentation, Konzentration, Parlamentsgröße - ab. Hält man eine bestmögliche Erfüllung des Repräsentationsziels für erstrebenswert, so ist die komplette Streichung einer Begrenzung von Ausgleichsmandaten zu empfehlen. Eine solche Reform wäre allerdings nicht nur in Bezug auf das Konzentrationsprinzip negativ zu bewerten, sondern wäre ebenfalls eine schlechte Lösung im Hinblick auf die Größe des Landtags.

Soll zusätzlich das Ziel erreicht werden, die tatsächliche Mandatszahl nicht allzu sehr von der gegenwärtig gesetzlichen von 69 abweichen zu lassen, dürfte kein Weg an einer größeren Reform des Wahlrechts vorbeiführen, die den Anteil der Direktmandate erheblich reduziert. Soll eine möglichst gute Repräsentation bei gleichzeitiger Begrenzung der Parlamentsgröße erreicht werden, so wäre eine Lösung zu empfehlen, die den Direktmandatsanteil verringert und Ausgleichsmandate beibehält. Ein kleiner Ausgleich würde gleichzeitig

25 In Nordrhein-Westfalen beispielsweise repräsentiert nach der letzten Landtagswahl 2010 ein direkt gewählter Abgeordneter durchschnittlich rund 100.000 Wahlberechtigte.

26 Eine solche technische Spezifikation ist auch nötig, wenn das Wahlrecht im Sinne eines großen Ausgleichs reformiert wird. Dass in dem hier konkret untersuchten Fall beim großen Ausgleich keine Unterschiede zwischen beiden technischen Versionen auftreten, lässt sich nicht verallgemeinern. Im Gegenteil: Genügt auch ein großer Ausgleich nicht zur Vollkompensation der Überhangmandate, so spielt auch dann die technische Ausgestaltung des Ausgleichs eine relevante Rolle. 
eine konzentrierende Wirkung entfalten und dem Ziel der kleinen Parlamentsgröße besser gerecht werden. Ob der große Ausgleich systematisch dem Repräsentationsziel besser Rechnung trüge, muss angesichts dieser Fallstudie kritisch hinterfragt werden.

Konzentration wäre am besten zu erzielen, wenn auf Ausgleichsmandate vollends verzichtet würde; hält man am Prinzip des Ausgleichs fest, müsste der kleine Ausgleich gewählt werden. Die diskutierten Vorschläge, die nicht danach trachten, Überhangmandate vollständig zu kompensieren und damit eine bestmögliche Repräsentation herzustellen, tragen damit natürlicherweise zur besseren Erfüllung der Konzentrationsfunktion bei, indem sie in der Regel die stärkste Partei begünstigen. Fasst man das zugrunde liegende Wahlsystem der personalisierten Verhältniswahl grundsätzlich als Verhältniswahlsystem auf, so ist dieser Effekt als Systemfehler zu interpretieren. Nimmt man die formale Konstruktion als Mischwahlsystem hingegen ernst, so ist positiv zu bewerten, dass bei grundsätzlicher Verfolgung des Repräsentationsziels auch ein Beitrag zur Konzentration geleistet wird. Ein Kompromiss in diesem Sinne könnte eine Reform darstellen, die 34 Direktmandaten 35 Listenmandate gegenüberstellt und einen kleinen Ausgleich vorsieht. Eine solche Regelung enthält eine konzentrierende Komponente bei gleichzeitiger Berücksichtigung des Repräsentationsprinzips und garantiert eine Obergrenze der Größe des Landtags. Die Aussichten, einen solchen Vorschlag gegen Alternativen mit Vollkompensation durchsetzen zu können, dürften allerdings entscheidend davon abhängen, inwieweit die gegenwärtig vorherrschende negative Bewertung von Überhangmandaten von einer differenzierten Sichtweise abgelöst wird. ${ }^{27}$

Sollte das reformierte Wahlrecht eine Regelung zur Begrenzung von Ausgleichsmandaten enthalten, so schlagen wir vor, die technische Ausgestaltung gemäß der hier diskutierten Version 1 zu übernehmen. Eine Regelung, die - wie durch Version 2 gegeben - eine Obergrenze an Mandaten vorsieht, sich aber gleichzeitig über diese Grenze hinwegsetzt, muss als paradox gelten.

Damit ist noch nicht gesagt, zu welchem Ergebnis die Reformbemühungen der Kieler Parlamentarier führen werden. Von außen lässt sich nur schwer ausmachen, welche Akteure sich welche der skizzierten Zielsetzungen zu eigen machen. Zur Prognose ihres Verhaltens könnte man zusätzlich das Eigeninteresse an einer möglichst guten Machtposition im Parlament ansehen. Dieses Argument spräche dafür, dass sich die CDU für eine Klarstellung des Landeswahlgesetzes im Sinne eines kleinen Ausgleichs einsetzen wird. Die anderen Parteien müssten demnach für eine Präzisierung im Sinne des großen Ausgleichs oder aber eine große Lösung plädieren, die die Entstehung von Überhangmandaten verhindert. Allerdings setzt diese Argumentation voraus, dass die Parlamentsakteure nur ihre momentane Interessenlage im Sinn haben. Das muss aber nicht der Fall sein, da kluge und erfahrene Abgeordnete erkennen dürften, dass sich ihre Interessenlage ändern und eine Reform sich gegen ihre Urheber wenden könnte. Beispielsweise profitierten die Sozialdemokraten bei den Landtagswahlen 1992 und 2000 von Überhangmandaten, während diese nun einer ihnen nicht willkommenen Regierung ins Amt verhalfen. Als weiterer Faktor ist die noch ausstehende Entscheidung des Landesverfassungsgerichts zum Wahlrecht zu berücksichtigen, da - dies lehrt die Erfahrung auf der Bundesebene - Verfassungsrichter in Wahlrechtsfragen als Initiatoren oder Vetomacht sehr einflussreich werden können. 